\title{
Research on Existing Problems and Countermeasures of Local Legislation on Traditional Chinese Medicine in China
}

\author{
Yuqing Zhang \\ College of Humanities \\ Hubei University of Chinese Medicine \\ Wuhan, China 430065
}

\begin{abstract}
The Law on Traditional Chinese Medicine" is the basic legal ground for local legislation on traditional Chinese medicine, and plays a significant role in promoting the development of traditional Chinese medicine in the new era. It at the same time shows the institutional space to improve local legislation. Local governments should seize the opportunity of implementing the law on traditional Chinese medicine and formulate local regulations that are targeted and effective according to the actual situation of the province. This paper summarizes the current situation and existing problems of local legislation of traditional Chinese medicine, and puts forward relevant suggestions centering on the basic principles of standardization, regionalism and nationality.
\end{abstract}

Keywords-traditional Chinese medicine; local legislation; standardization; nationality

\section{INTRODUCTION}

In July 2017, the general office of the former National Health and Family Planning Commission and the Office of the State Administration of Traditional Chinese Medicine issued the "Notice on Strengthening the Local Regulations and System Construction of Traditional Chinese Medicine". It required all regions to review existing local laws and regulations related to traditional Chinese medicine, timely put forward suggestions on the clean-up, and promote the establishment, revision, abolition and interpretation of the law, so as to ensure the unity and authority of "The Law on Traditional Chinese Medicine".

\section{BASIC INFORMATION OF LOCAL CHINESE MEDICINE LEGISLATION IN CHINA}

\section{A. Overall Situation}

In addition to Hong Kong, Macao and Taiwan, 25 of the 31 provinces (autonomous regions and municipalities directly under the central government) have enacted local laws and regulations on traditional Chinese medicine (TCM), accounting for $80.6 \%$ of the total. By February 2018, 29 provinces (autonomous regions and municipalities directly under the central government) had formulated or revised local regulations. Hebei took the lead in promulgation of the revised "The Regulation on Traditional Chinese Medicine in
Hebei Province", and Beijing, Shanghai, Hubei, Heilongjiang and other provinces and municipalities directly under the central government have formally included the work in the legislative plans or research projects of local people's congresses and government legislative affairs offices.

\section{B. Characteristic Samples}

1) "Regulations of Shenzhen Special Economic Zone on Traditional Chinese Medicine" (hereinafter referred to as "Shenzhen Regulations") (2010): As the first local regulation of traditional Chinese medicine after the implementation of the new medical reform in China, and also the first regulation of traditional Chinese medicine among the 15 sub-provincial cities, "Shenzhen Regulation" is a significant achievement in the implementation of the new medical reform in Shenzhen, and also the core achievement of building a strong province in terms of traditional Chinese medicine in Guangdong. The Shenzhen Special Economic Zone took the lead to promote the licensing and establishment of traditional Chinese medicine centers and medical institutions, as well as the research, formulation and promulgation of supporting documents and policies related to master-apprentice education, key disciplines, famous doctors, famous departments and medical quality assessment. In this way, Shenzhen's traditional Chinese medicine characteristics and advantages are more prominent, and the traditional Chinese medicine of Guangdong has achieved the important goal of "small changes in three years and a major change in five years".

2) "Regulations on the Protection of Chinese Herbal Medicines in regions south of the Five Ridges, Guangdong Province" (2016): The introduction of the regulation is a local legislation case showing the courage to practice and innovation. Released in the "gap" when "The Law on Traditional Chinese Medicine" had yet to be enacted, it not only has typical practical value to the creation of special law of local legislation of traditional Chinese medicine, but also provides a useful attempt to answer the proposition of "how 
to implement local legislation in the absence of superior law". The regulation puts forward the first batch of protection of Chinese medicinal materials (8 kinds of Chinese medicinal materials, such as exocarpium, citrus chachiensis hortorum, fructus amomi villosi and agilawood), and clearly stipulates the construction standards and legal responsibilities of provenance, planting and brand protection of Chinese medicinal materials in regions south of the Five Ridges.

3) "The Regulation on Traditional Chinese Medicine in Hebei Province" (hereinafter referred to as "Hebei Regulation") (2018): "Hebei Regulation" is the first local regulation on traditional Chinese medicine to be revised after the implementation of the law on traditional Chinese medicine. Adhering to equal emphasis on promotion and standardization, the regulation lives up to the principles of "making the spirit of major laws concrete, turning policy orientation into measures, abstracting the provincial experiences to statutes and localizing nonlocal practices" [1], so as to solidify important policies and measures to support and promote the law of traditional Chinese medicine.

\section{MAIN PROBLEMS EXISTING IN LOCAL LEGISLATION of TRADITIONAL CHINESE MEDICINE}

\section{A. Legislation and Amendments Are Not Timely, Lagging Behind the Legal Needs of TCM}

With the gradual improvement of China's TCM policy system, the TCM medical service system has also been gradually established. However, the problems in the development of local TCM are increasingly prominent, which are mainly reflected in the shortcomings of the governance model and the structural defects of the management system. The most fundamental reason is the insufficient understanding of the positioning and role of TCM. There are many policies and conceptual provisions and few operational provisions, making it difficult to comprehensively plan and coordinate the development of TCM. At present, 12 of the 25 provinces (autonomous regions and municipalities directly under the central government) that have enacted local laws and regulations have not revised them, accounting for $48 \%$ of the total. The legislation in each of these 12 provinces (autonomous region, municipality directly under the central government) Shanghai (enacted its laws and regulations in 1991), Beijing (in 2001), Anhui (in 2001), Gansu (in 2001), Qinghai (in 2002), Shanxi (2002), Hubei (2002) and Ningxia (2002), was over 16 years. The laws and regulations are antiquated and lack a positive response to the legal demand for the development of TCM, seriously hindering the development of local TCM.

\section{B. The Content of Laws and Regulations Is Out-of-balance, and There Is a Lack of Institutional Coordination Between Traditional Chinese Medicine and Traditional Chinese Medicinal Materials}

Since ancient times it is clear that "medicine cannot be separated from drug" and medicine and drug are of the same origin. For a long time, traditional Chinese medicine and traditional Chinese medicinal materials have been divided artificially due to the reasons of management system. In many local regulations, the word "药" (drug, here referred to as traditional Chinese medicinal materials) is deliberately omitted from their names. Among the 25 provinces (autonomous regions and municipalities directly under the central government) that have promulgated local Chinese medicine (traditional Chinese medicinal materials) regulations, there are 16 which haven't mention the expression "traditional Chinese medicinal materials" in the names of regulation, mostly expressed as "development of traditional Chinese medicine regulations, which accounts for $64 \%$ of the total; there are 8 that used the expression as "regulations on Chinese medicine and traditional Chinese medicinal materials, accounting for $32 \%$, of which the regulations of Shanxi, Henan, Sichuan, Yunnan, Hebei and Heilongjiang are reformulated ones after the original regulations were repealed.

In addition, in terms of the use and management system of traditional Chinese medicinal materials, except for the special provisions on the medicinal materials of ethnic minorities in ethnic autonomous areas and the mention of some provinces in the TCM service system, most of the local legislation has few provisions.

\section{The Legislative Pattern Is Limited, Putting Too Much Emphasis on the Standard of TCM Service Industry}

Due to the long-term absence of the basic law of medical and health care and the law of traditional Chinese medicine, the local legislation of traditional Chinese medicine has great historical limitations. It usually regards the Chinese drug as a matter of medical care. Therefore, some early local laws and regulations are closer to the "TCM management law", which focuses more on the industry management centering on medical institutions and personnel and less on the system design that reflects the characteristics of TCM and local characteristics. For example, health preservation, tourism, education, culture and other elements have not been integrated into the institutional framework of the development of traditional Chinese medicine. At best, there are some basic, incentive or policy legislative expressions about them. If local laws and regulations of traditional Chinese medicine are designed only according to the industry thinking of medical service, there will be a "fault" with the legislative thought determined by the current strategic positioning of traditional Chinese medicine [2].

\section{THE BASIC PRINCIPLES TO BE FOLLOWED IN LOCAL LEGISLATION OF TRADITIONAL CHINESE MEDICINE}

\section{A. The Principle of Standardization}

1) Strict and standard structure and legal terms:

"The Law on Traditional Chinese Medicine" is structured by three parts: general provisions, sub-provisions and supplementary provisions. The sub-provisions are clearly made up of seven parts, namely, TCM service, TCM protection and development, TCM talent training and legal responsibility, etc. There are mainly two modes of local 
regulations on traditional Chinese medicine. The first can be divided to three parts of general provisions, sub-provisions and supplementary provisions as "The Law on Traditional Chinese Medicine". In the other mode, all the clauses are piled up together, with no logic between the style structure and incomplete content. For example, with 31 clauses, no chapters have been specially divided in the "Regulations on the Development of Traditional Chinese Medicine in Guizhou Province", and this is also true in "Regulations on the Development of Traditional Chinese Medicine in Guangxi Zhuang Autonomous Region".

Local legislation should first ensure logical and rigorous structure; second, it should make the content of regulations well-bedded and definite; third, it should ensure the standardization and certainty of legal terms and try to avoid using ambiguous concepts.

2) The principle of "non-contravention": Local legislation of traditional Chinese medicine should follow the general guiding principles of legislative activities, such as the principles of democracy and science, and ensure the unity and coordination of legal systems. It shall not contravene the constitution, laws, administrative regulations of the state council and local regulations of each province. At the same time, local legislation of traditional Chinese medicine has its own particularity and special principles. To be more specific, first, it is necessary to investigate and find out the specific local situation; second, it is necessary to be familiar with relevant laws and regulations; third, it is necessary to good at summarizing and grasping the special contradictions and problems that need to be solved by the local laws and regulations of TCM; and fourth, it is necessary to maintain a prudent attitude at all times, be insufficiently mature in terms of conditions, and avoid making rash decisions on problems that are contradictory or controversial between parts and the whole. Only in this way can practical decisions be made and the authority and seriousness of law be maintained.

3) Standardizing legislation and attaching importance to the quality of legislation: The vitality and authority of local laws and regulations lie not in quantity, but in quality, that is, whether they can solve practical problems. First of all, it is needed to standardize the legislative act itself. Efforts should be made to make the local legislation linked up with state laws and administrative regulations, local laws and regulations linked up with each other and local laws and regulations linked up with administrative rules so as to make the legislative act feasible and practical. Second, democracy and transparency of legislation should be enhanced to ensure the legality and fairness of legislation of TCM. Third, it is also needed to improve the prediction and response capacity of local legislation, so that it can play a leading role.

\section{B. The Principle of Locality}

The so-called locality refers to "starting from the specific situation and actual needs of the locality, stipulate what needs to be stipulated so as to make the local laws and regulations targeted and operable, and really promote and guarantee reform, development and stability in local places" [3]. As a specific practice to respond to and implement the rule of law construction of traditional Chinese medicine, it is necessary for local legislation to proceed from "local conditions" and "people's conditions" to solve the special local problems in the development of traditional Chinese medicine.

1) Paying attention to "industry thinking": Local legislation of traditional Chinese medicine should not be limited to the thinking mode of "medical service", but should be accommodated to the "industry thinking". Efforts should be made to expand TCM services, upgrade the TCM industry, and integrate TCM with old-age care, tourism, and cultural industries. At the same time, it is necessary to pay attention to the boundary issue, which is to avoid the erosion of the public welfare of TCM by "excessive marketization".

2) Revitalizing the medical services of ethnic minorities: "Traditional Chinese medicine is a general term for all ethnic medicines in China, including those of the Han nationality and ethnic minorities". In the course of their long-term survival and development and medical practice, various ethnic groups in China have formed their own ethnic medicine systems, such as Mongolian medicine, Tibetan medicine, Zhuang medicine, Uygur medicine and Hui medicine. Experiencing a long history of development, they are all the treasure house of traditional Chinese medicine and an important part of excellent national culture, with distinct national characteristics, regional characteristics and unique theoretical system and clinical characteristics.

a) Legislative status and existing problems: China has five ethnic autonomous regions, 30 ethnic autonomous prefectures and 120 ethnic autonomous counties (banners). Apart from Tibet and Xinjiang, the other three ethnic autonomous regions have promulgated local laws and regulations, namely, "The Regulations on the Development of Traditional Chinese Medicine in Ningxia Hui Autonomous Region", "The Regulations on the Development of Traditional Chinese Medicine in Guangxi Zhuang Autonomous Region", and "The Regulations on the Development of Traditional Chinese Medicine in Inner Mongolia Autonomous Region". In addition, in 2001, Gannan Autonomous Prefecture of Gansu issued "Regulations on the Development of Tibetan Medicine in Gannan Tibetan Autonomous Prefecture of Gansu"; in 2005, Fuxin Mongolian Autonomous County of Liaoning Province issued "The Regulations on the Administration of Mongolian Medicine in Fuxin Mongolian Autonomous County; in 2009, the Korean Autonomous Prefecture of Yanbian issued "The Regulations on the Development of Korean Medicine in Yanbian Korean Autonomous Prefecture"; and in 2009, the Xiangxi Tujia and Miao Autonomous Prefecture of Hunan province issued "The Regulations on the Protection of Tujia Medicine and Miao 
Medicines of Tujia medicine in Xiangxi Tujia and Miao Autonomous Prefecture".

In other provinces with relatively large concentrations of ethnic minorities, the contents of ethnic medicine are included in the local laws and regulations on traditional Chinese medicine. In "Regulations on the Development of Traditional Chinese Medicine in Gansu Province" a special chapter is set on "ethnic medicine". Yunnan, Guizhou and Jilin provinces have provisions on the cultivation of ethnic medical personnel and the establishment of ethnic medical institutions, and encourage ethnic autonomous areas to formulate separate regulations.

There are also some problems in the legislation of minority medicine. First, it is difficult for the decentralized legislative model, with a lower level to reflect and guarantee the important status and development of minority medicine. Second, the legal provisions are mostly macro policy provisions, with a lack of specific operational provisions. Third, the characteristics of traditional medicine of ethnic minorities are ignored. Four, there is an imbalance between the protection of public law and the protection of private law, and there is a lack of specific and clear provisions on the protection of property rights and benefit sharing mechanism of ethnic minority medical resources.

b) Countermeasures and suggestions: Attention should be paid to the following points in local legislation on traditional Chinese medicine to reflect local characteristics and revitalize the ethnic minority medicine:

- to maintain and develop the characteristic advantages of ethnic minority medicine and follow their own development rules and characteristics;

- to establish the key protection and inheritance mechanism of traditional medical knowledge of ethnic minorities;

- to clarify the identification conditions and procedures of folk physicians of ethnic minorities;

- to clarify the ecological protection mechanism of ethnic minority traditional medicine genetic resources;

- to clarify and standardize the relief approach of the subject's rights, and pay attention to the convergence with the existing law.

\section{The Principle of Nationality}

1) The nationality of traditional Chinese medicine

TCM is the medical system of the Chinese nation. After thousands of years of development, theories of yin-yang and five elements, viscera and meridians, and collaterals, qiblood-body fluid, etiology and pathogenesis have taken shape, forming a theoretical system with unique characteristics of TCM [4]. It has a set of philosophical thoughts and ways of thinking, which embody the view of nature, epistemology and methodology of the Chinese nation. At the same time, traditional Chinese medicine has played an important role in the endless development of the Chinese nation. The nationality of TCM is embodied in the following aspects:

a) The cornerstone of TCM is the "Chinese" way of thinking: In the historical process of human development, the way of thinking is a process of development and change, but for the national way of thinking, it is not easy to change. Traditional Chinese medicine is a typical science that embodies the characteristics of traditional Chinese thinking. One of the characteristics is the holistic view of "harmony between man and nature" Starting from the holistic view of "harmony between man and nature", to learn the essence of things is 'image' abstracting, and the intuition or instinct, introspection or perception in the shifting and flowing 'images', are shown as the process of irrational thinking" [5] The second is the everlasting view of dynamic balance. Different from the western way of thinking, the Chinese attaches great importance to the overall balance while emphasizing the opposition of Yin and Yang. The yin-yang theory is the theoretical foundation of traditional Chinese medicine, which holds that Yin and Yang are peaceful between heaven and earth. In addition, diagnosis, syndrome differentiation, prescription, medication, acupuncture, qigong, Tuina and other techniques and methods of TCM are also under the "Chinese" conception of thinking with strong national characteristics.

b) Ethnic culture is the soil for the growth of traditional Chinese medicine: The traditional medical knowledge of each nation depends on folk culture and religious tradition, and carries the group wisdom and national spirit of the nation. "There is hardly any other science in the world today that can so tenaciously maintain the traditional disciplinary system with national cultural characteristics as medicine." "Medicine has existed as a survival technique at first in history, so it must have a broad basis of public awareness and practice. [6]" Traditional Chinese medicine is a national medical method, which is related to the geographical environment and national characteristics and reflects the specific customs and religious cultural background of each ethnic group.

2) Inheritance and innovation of nationality

a) Building a traditional Chinese medicine inheritance system with national characteristics: The inheritance of TCM is the inevitable course "to inherit the sage's knowledge". The current situation of academic inheritance of traditional Chinese medicine is not optimistic, which is mainly reflected in the problems including unclear main duties and responsibilities of the government, the lack of emphasis on academic inheritance, and the insufficient of standardization in academic inheritance projects and inheritor selection, etc. Attention should be paid to standardize the following aspects.

First, it's necessary to define the specific responsibilities of governments at or above the county level. For example, article 43 of "The Regulation on Traditional Chinese Medicine in Shenzhen Special Economic Zone" stipulates the government's obligation to subsidize inheritance. 
Second, it's necessary to clarify the content and mode of inheritance. Although some provinces have stipulated that provincial health administrative departments should establish and formulate TCM inheritance system, there is a lack of further clear definition of the famous academic materials of traditional Chinese medicine, characteristic traditional Chinese medicine technology, academic thoughts and diagnosis and treatment experience as the objects of inheritance, which make the inheritance activities disorderly and extensive.

Third, it's necessary to protect the legitimate rights and interests of academic inheritors of traditional Chinese medicine in accordance with the law. Through "empowering" inheritors, local legislation can balance the rights (power) structure between inheritors and the government, and build a "benefit sharing" inheritance system featuring rights protection, so as to realize multiple rights protection and meet the demands of people's livelihood.

b) Strengthening the knowledge protection and technology mining of traditional Chinese medicine: Traditional Chinese medicine knowledge includes theoretical knowledge of traditional Chinese medicine, traditional Chinese medicine prescription, diagnosis and treatment technology, traditional Chinese herb resources related to traditional Chinese medicine knowledge, processing technology of traditional Chinese medicine, unique symbols of traditional Chinese medicine and so on. Created and handed down from generation to generation, it is the sum of knowledge, skills and practices based on theories, beliefs and experiences unique to traditional Chinese culture, and it is the medical and health knowledge with social and economic value. In the legislation of local traditional Chinese medicine, it is necessary to pay attention to the protection and technical exploitation of traditional Chinese medicine knowledge in the region, actively use the intellectual property system to complete the strategic layout, and complete the inheritance and innovation of traditional Chinese medicine knowledge.

c) Actively promoting innovation in TCM theories: To strengthen the inheritance and innovation of TCM theories, efforts should be made through multiple channels, including the following aspects.

- It is necessary to adhere to the principle of "TCM theory should be confident, the inheritance and innovation should be conscious and rules should be set up for self-reliance".

- It is necessary to adhere to the combination of theory and practice. Efforts should be made to give full play to the advantages of traditional Chinese medicine to solve the health problems of the people and enhance the guiding value of theory for clinical and scientific research.

- It is necessary to actively carry out interdisciplinary research. Multidisciplinary techniques and methods should be adopted to refine new research ideas and seek new development opportunities.
- It is necessary to accelerate the construction of discipline platform. Efforts should be made to intensify efforts to build national and provincial TCM theory inheritance and innovation bases, actively build key research institutes (offices) for TCM theory, and set up national collaborative innovation centers.

\section{SEVERAl Key IsSUES That SHOULD Be PAID ATTENTION TO IN LOCAL LEGISLATION OF TRADITIONAL CHINESE MEDICINE}

In the "post-TCM law period", local governments should avoid the "comprehensive revision" mode which is extensive. It is necessary for them to adopt the precise legislative mode of "solving problems", and "crush one by one" the individualized problems in the development of traditional Chinese medicine, so as to solidify some policies and measures to support and promote TCM law in the legal situation.

\section{A. Raising Awareness of the Importance of Legislation on TCM}

With the advantages and role of the "five" resources of traditional Chinese medicine, to ensure the development of traditional Chinese medicine through legislation is a major measure for China's economic and social development. Local governments should enhance their sense of responsibility and mission, fully and accurately grasp the essence and basic contents of the law on traditional Chinese medicine, and actively legislate to amend the law in light of local conditions, so as to provide strong legal guarantee for the revitalization of the cause of traditional Chinese medicine.

\section{B. Improving the Supervision and Law Enforcement System of Local TCM Laws and Regulations}

1) Establishing strict measures and procedures for TCM supervision and law enforcement: First, it is necessary to focus on the three main lines of supervision: institutional supervision, personnel supervision and technical supervision second, it is necessary to clarify the responsibilities and management institutions of the government, establish and improve the coordinated law enforcement mechanism; third, it is necessary to establish a comprehensive supervision system (administrative supervision, industry self-discipline, social supervision, public participation); fourth, it is necessary to adopt differentiated supervision mode in which different modes are used according to different subjects; finally, it is necessary to establish a regulatory information system, a bad practice record system, a negative list system and a joint punishment mechanism for dishonesty.

2) Refining the relevant systems of industry associations: In local traditional Chinese medicine legislation, the regulations on the participation of industry associations in industry governance are weak, and most of them are basic provisions and are of low operability. The relevant system of industry association should be refined to legalize its functions. First, it is necessary to clarify the legal status and 
legal functions of TCM industry associations; second, governments at all levels should strengthen their guidance and construction of TCM expert committees, TCM industry associations, and societies of integrated traditional Chinese and western medicine; third, it is necessary to establish an industry integrity file and a risk warning mechanism, and strengthen self-regulatory oversight of the industry.

\section{Paying Equal Attention to Traditional Chinese Medicine and Traditional Chinese Medicine and Traditional Chinese Herbs, and Using High-quality Authentic Chinese Medicinal Materials to Ensure the Realization of Magical Curative Effect of Traditional Chinese Medicine}

The vitality of TCM lies in traditional Chinese herbs. As the material basis for the inheritance and development of traditional Chinese medicine, Chinese herbal medicines is a strategic resource related to national economy and people's livelihood, but it is also facing severe challenges. The production technology of Chinese herbal medicines lags behind, with an orientation of overvaluing yield while undervaluing quality. Abuse of chemical fertilizers, pesticides, growth regulators is widespread, and adulteration, doping, staining, sulfur fumigation, heavy metals and other problems are prominent. Articles 21-25 of "The Law on Traditional Chinese Medicine" specify specific measures for the protection and development of Chinese herbal medicines, mainly involving quality standards, quality testing and traceability system, etc. "The Law on Traditional Chinese Medicine" defines "geo-authentic crude drug" from the legal level for the first time. The formation of geo-authentic crude drug is related to the unique geographical and cultural background of China and the theory of traditional Chinese medicine. The culture of geo-authentic crude drug contains a lot of natural, humanistic and social knowledge, which is one of the sources of modern medicine innovation and one of the decisive factors of traditional Chinese medicine culture inheritance and development. Therefore, "protecting the resources of geo-authentic crude drug and ensuring their quality and safety" should be the focus of the whole system design of local legislation.

\section{CONCLUSION}

"In a sense, the construction of the rule of law in a modern country is the combination of the general and macroscopic national rule of law and the concrete and subjective local rule of law. The concreteness and subjectivity of the rule of law are mainly reflected by the local rule of law". [7] The construction of local legislation is conducive to timely response and treatment of regional and individual problems in the development of local traditional Chinese medicine, so as to achieve the nationwide improvement of traditional Chinese medicine legal system.

\section{REFERENCES}

[1] Shen Zhengxian, Tian Yanxun. The Regulation on Traditional Chinese Medicine in Hebei Province [N]. China Journal of Traditional Chinese Medicine, 2017, 12-06. (in Chinese)
[2] Zhang Boyuan. Practical difficulties and system construction of local legislation of traditional Chinese medicine [J], Local Legislation Journal, 2018 (3): 116

[3] Liu Yungeng. Benign Legislation for the People: Reflections on Further Strengthening and Improving Local Legislation [J]. Shanghai Municipal People's Congress, 2011 (2): 7 (in Chinese)

[4] Liu Guowei, Zuo Ning. An Analysis of the Features of TCM Intercultural Communication History [J]. Clinical Journal of Chinese Medicine, 2014 (15): 2. (in Chinese)

[5] Wang Shuren, Yu Bolin. Rediscovery of Traditional Wisdom (part 2) [M]. Beijing: The Writers Publishing House, 1996: 199. (in Chinese)

[6] Qiu Hongzhong. Medicine and Human Culture [M]. Changsha: Hunan Science \& Technology Press, 1993: 3-564. (in Chinese)

[7] Feng Lixia. Take the Rule-of-law at Local Level Seriously - In the Perspective of the Function of Local Legislation in State-Building [J]. Research on local legislation, 2016 (1): 1. (in Chinese) 